\title{
Particle-grain boundary interactions: A phase field study
}

Karim Ahmed ${ }^{1 *}$, Michael Tonks ${ }^{2}$, Youngfeng Zhang ${ }^{1}$, Bulent Biner ${ }^{1}$, and Anter El-Azab ${ }^{3}$

1. Fuel Modeling and Simulation Department, Idaho National Laboratory, Idaho Falls, ID, USA

2. Department of Mechanical and Nuclear Engineering, Pennsylvania state University, State College, PA, USA

3. School of Materials Engineering, Purdue University, West Lafayette, IN, USA

E-mail: karim.ahmed@inl.gov

Abstract. A detailed phase field model is used to investigate particle-grain boundary interactions. The model takes into consideration both the curvature-driven grain boundary motion and particle migration by surface diffusion. The phase field model relaxes all the restrictive assumptions usually employed in the classical and sharp-interface models of the problem. Our 2D and 3D simulations demonstrate that the model captures all possible particle-grain boundary interactions proposed in theoretical models and observed in experiments. For high enough surface mobility, the particles move along with the migrating boundary as a quasi-rigid-body (i.e., without change in shape or size), albeit hindering its migration rate as compared to the particle-free case. For less mobile particles, the migrating boundary can separate from the particles. For the case of steady-state motion of the particles with the migrating boundary, evolution equations for the grain size were derived that predict a strong dependence of the grain growth rate on the number of particles, particle size, and surface diffusivity. For the case of boundary breakaway, the separation condition was found to agree well with predictions from theoretical calculations. However, our results show that non-uniform particle distribution promotes boundary detachment. The results also demonstrate that it is easier for a migrating boundary to separate from a spherical particle than from a cylindrical particle, and hence 2D simulations underestimate boundary breakaway.

Keywords: Grain growth; Particle-grain boundary interaction; Phase field modeling; Sintering 


\section{Introduction}

All physical properties of polycrystalline solids such as yield stress, fracture strength, electrical breakdown strength, dielectric constant, and thermal conductivity are strongly dependent on the grain size [1-3]. This grain size dependence is due to the role grain boundaries play in influencing materials properties. Moreover, controlling grain growth is crucial for achieving the desired density for ceramics during sintering $[1,2]$. The densification rate for these materials is lower for larger grain size [1]. Furthermore, it was found out that the performance of several materials under extreme conditions, such as irradiation, high temperature, and high stresses is highly affected by the grain size [1-4]. For polycrystalline solids containing second-phase particles such as inclusions, precipitates, pores, or bubbles, the interactions between these particles and the migrating grain boundaries determine the overall grain growth rates in such materials $[1,5-15]$. Therefore, a good understanding of the particle-grain boundary interactions is essential for obtaining accurate grain growth rates in real systems.

Second-phase particles exert a drag force on a migrating grain boundary, which retards the grain growth process $[1,5-15]$. Several theoretical models were proposed to study the so-called particleinhibited grain growth [1,5-15]. These models can be divided into two classes depending on whether the particles are considered mobile or immobile. Immobile particles (e.g., inclusions or precipitates) may pin the boundary completely and stop grain growth as was first predicted by Zener [1, 15-17]. By contrast, mobile particles (e.g., pores or bubbles) can be dragged along with a migrating boundary as long as one or more of the matter transport mechanisms such as surface diffusion, bulk diffusion, or evaporation and condensation is active [1, 5-14]. Nonetheless, a migrating grain boundary with high enough velocity can break away from mobile or immobile particles [1, 5-16].

For the case of immobile particles, Zener derived a relation for the limiting grain size (the maximum grain size that can be attained during grain growth) as function of the particle radius and volume fraction $[1,15-17]$. Several investigations were conducted later to refine Zener's relation by relaxing some of his 
model assumptions [1, 15-17]. On the other hand, modeling the effect of mobile particles on grain growth proved more difficult $[1,5-14]$. In such case, in addition to the grain boundary motion, the particle migration via matter transport must be accounted for. Nichols [5], Brook [6], and Carpay [7] were the first to introduce such models. In these classical models, the particle and boundary shapes were assumed to be spherical. Moreover, these models assume that the particle move along with the boundary as a rigid-body (e.g., without change of its shape or size). Furthermore, they only treated homogeneous microstructures where the migration of only one particle-boundary complex can be considered to be a representative of the average kinetics of the whole system. More sophisticated models then emerged to alleviate the shortcomings of the classical models [8-13]. In these models, which are called sharp-interface models, the grain boundary is assumed to move under the influence of its curvature, while particle migration take place via surface diffusion. Velocity equations for segments of the grain boundary and particle surface are then derived and supplemented by appropriate boundary conditions at the particle tips. The sharpinterface models provided more insight about the interactions between mobile particles and grain boundaries. For instance, it was demonstrated that a particle on a triple junction retards the grain growth process more than a particle on a two-grain junction [12]. It was also shown that it is much easier for a migrating boundary to detach form a spherical particle than from a cylindrical particle [8-12]. However, solving these sharp-interface models numerically for general 3D particle and grain shapes is a cumbersome task [8-13].

The numerical difficulty of solving the sharp-interface models and limitations of the classical models motivated us to introduce a diffuse-interface (phase field) description of the problem [18-21]. Since explicit tracking of surfaces/interfaces is not required in these models, general 3D particle and grain geometries can be handled in a straightforward manner. While our earlier studies only considered the effect of particles on the average kinetics of grain growth in a polycrystalline solid with attention to the average grain growth rates and exponents as function of the particle volume fraction, we focus here on the 
details of particle-grain boundary interactions. Specifically, we investigate both the steady-state motion of the particles with the migrating boundary and the condition for boundary separation from the particles. We also study the effect of particle size and morphology on the grain boundary migration rate. Moreover, for a few idealized 2D and 3D grain and particle shapes, we derive analytical grain growth laws that reflect the effect of particle drag. The results from our 2D and 3D simulations agree well with the derived growth laws. Furthermore, in contrast to our earlier investigations where explicit finite difference scheme was used to solve the phase field kinetic equations [18-20], we utilize here a fully-coupled, fully-implicit finite-element scheme that was implemented via the phase field code MARMOT developed at Idaho National Laboratory [22].

This manuscript is organized as follows. First, the theoretical models of particle-grain boundary interactions are reviewed in section 2. We then summarize the basic features of the phase field model in section 3. The results are presented in section 4. In section 5, we draw the main conclusions and discuss some possible future extensions of the work.

\section{Theoretical models of particle-grain boundary interactions}

Second-phase particles exert a drag force on a migrating boundary that hinders its velocity. In order to investigate the so-called particle-inhibited grain growth, a few theoretical models were proposed in literature [1, 5-14]. These models can be classified into two categories depending on whether the particles are treated as mobile or immobile. Second-phase particles such as inclusions or precipitates are usually considered immobile, while pores and bubbles are often considered mobile.

\subsection{Immobile particles}

The effect of immobile particles on the grain growth process was first studied by Zener [1]. He considered the particles to be immobile, spherical, mono-size, insoluble, and randomly distributed in the polycrystalline solid. Zener considered the curvature to be the driving force for the boundary motion. The 
presence of the particles exerts a drag force on the boundary that hinders its motion. The velocity of the boundary is then given by

$$
v_{\mathrm{b}}=M_{\mathrm{b}}\left(F_{\mathrm{b}} \quad N_{\mathrm{p}} F_{\mathrm{p}}\right)
$$

where, the subscript $\mathrm{b}$ denotes the boundary and $\mathrm{p}$ denotes the particle, $v_{\mathrm{b}}$ is the grain boundary velocity, $M_{\mathrm{b}}$ is its intrinsic (particle-free) mobility, $F_{\mathrm{b}}$ is its intrinsic driving force, $N_{\mathrm{p}}$ is the number of particles per grain boundary area, and $F_{\mathrm{p}}$ is the drag force exerted by the particle on the boundary. In the absence of the particles, the intrinsic driving force of the boundary due to its curvature is

$$
F_{\mathrm{b}}=\frac{\mathrm{b}}{R}
$$

Here, is a geometric factor that equals 2 for a spherical grain, $\quad{ }_{\mathrm{b}}$ is the grain boundary energy, and $R$ is the grain radius. The particle drag force depends on the particle shape [14]. For a spherical particle, it is given by

$$
F_{\mathrm{p}}=2 r_{\mathrm{b}} \cos \sin ,
$$

where $r$ is the particle radius, and is the drag angle. For a cylindrical particle, the drag force per unit length of the boundary is

$$
F_{\mathrm{p}}=2_{\mathrm{b}} \sin ,
$$

According to Eq. (1), there are two possible scenarios for the interaction between an immobile particle and a grain boundary. First, the boundary can break away and leave the particle behind if $F_{\mathrm{b}} \square N_{\mathrm{p}} F_{\mathrm{p}}$. Second, the particle can completely pin the boundary and stop the grain growth if $F_{\mathrm{b}}=N_{\mathrm{p}} F_{\mathrm{p}}$. 


\subsection{Mobile particles}

The effect of mobile particles such as pores or bubbles on the kinetics of grain growth is more complicated. A migrating boundary exerts a force on the particle causing it to changes its shape, which could lead to matter transport from the leading surface of the particle to the trailing surface. Therefore, the particles can easily be dragged along by the moving grain boundary [1,5-14] if one or more of the matter transport mechanisms such as evaporation and condensation, surface diffusion, and lattice (volume) diffusion is active. The first models to investigate the effect of mobile particles on grain growth were proposed by Nichols [5], Brook [6], and Carpay [7]. In these simplified models, the microstructure is assumed to be homogeneous. Hence, only one particle-boundary complex is used to represent the behavior of the whole system. Moreover, the details of the grain boundary and particle shapes and nature of motion are ignored. According to these models, there are two different scenarios for the interaction between a mobile particle and a grain boundary. In one case, the migrating boundary could separate from the particle. In the other, the migrating boundary could drag the particle along with it. If the boundary separates from the particle, the boundary moves with its intrinsic velocity as in the particle-free case.

Boundary breakaway will simply occur whenever the grain boundary velocity $v_{\mathrm{b}}$ exceeds the particle velocity, $v_{\mathrm{p}}$. This condition can be expressed as [1, 5-7],

$$
M_{\mathrm{p}} F_{\mathrm{p}}<M_{\mathrm{b}}\left(F_{\mathrm{b}} \quad N_{\mathrm{p}} F_{\mathrm{p}}\right)
$$

In the above, $M_{\mathrm{p}}$ is the particle mobility. The forces have the same expressions as in Eqs. (2-4).

Rearranging Eq. (5), the boundary separation (breakaway) condition is expressed as,

$$
F_{\mathrm{b}}>N_{\mathrm{p}} F_{\mathrm{p}}+\frac{M_{\mathrm{p}} F_{\mathrm{p}}}{M_{\mathrm{b}}} .
$$

On the other hand, if the particle moves along with the boundary, the velocity of the particle-boundary complex $(v)$ can be obtained, by rearranging Eq. (5) and noting that $v=F_{\mathrm{p}} / M_{\mathrm{p}}$, as $[1,5-7]$ 


$$
\begin{aligned}
& v=M^{\text {eff }} F_{\mathrm{b}}, \\
& M^{\text {eff }}=\frac{M_{\mathrm{p}} M_{\mathrm{b}}}{M_{\mathrm{p}}+N_{\mathrm{p}} M_{\mathrm{b}}} .
\end{aligned}
$$

In the above, $M^{\text {eff }}$ is the effective mobility of the particle-boundary complex. Two limiting cases are immediately obtained from Eq. (7). When $M_{\mathrm{p}} \square N_{\mathrm{p}} M_{\mathrm{b}}$, the effective velocity of the particle-boundary complex reduces to $v=M_{\mathrm{b}} F_{\mathrm{b}}$, hence the effect of particles on the boundary velocity is negligible, a case which is commonly referred to as boundary-controlled grain growth. The other limiting case is when $M_{\mathrm{p}} \square N_{\mathrm{p}} M_{\mathrm{b}}$. In this case, the velocity of the particle-boundary complex becomes $v=M_{\mathrm{p}} F_{\mathrm{b}} / N_{\mathrm{p}}$, and hence the boundary velocity is limited by the particle mobility. This case is referred to as particlecontrolled grain growth. If one ignores particle coarsening and densification (e.g., during sintering) and assume the particle move along with the boundary as a rigid-body, expressions for the particle mobility can be derived for idealized particle shapes for different migration mechanisms [1, 5-13]. Therefore, in such situations, Eq. (7) can be directly integrated to arrive at the grain growth law as will be shown later. In the classical homogeneous models summarized above, it was assumed that the microstructure is homogeneous, and the details of the particle and boundary shapes and nature of motion were neglected. In order to alleviate these shortcomings, more advanced models were proposed [8-13, 18-21]. The first are so-called sharp-interface models [8-13]. In that description, the grain boundary moves by mean curvature while the particle migrates via surface diffusion. Surface diffusion is expected to be the dominant mechanism of particle migration in solids at temperatures of interest [1]. The shrinkage of particles (e.g., densification) is ignored and hence the particles have a prescribed constant volume.

The sharp-interface dynamics of the co-evolution of particles and grain boundary can be summarized as follows. The grain boundary moves under the influence of its curvature according to the relation,

$$
v_{b}={ }_{\mathbf{b}} M_{b} b .
$$


Here, $\quad b$ is the grain boundary local curvature (mean curvature in 3D). The curvature is positive for convex surfaces and negative for concave surfaces. On the other hand, the particle moves via surface diffusion as

$$
v_{\mathrm{p}}=\frac{{ }_{\mathrm{s}} D_{\mathrm{s} s}}{k_{\mathrm{B}} T} \nabla_{\mathrm{s}}^{2} \mathrm{~s}
$$

In Eq. (9), $v_{\mathrm{p}}$ is the velocity of a surface element of the particle, $\mathrm{s}_{\mathrm{s}}$ is the particle surface energy, $D_{\mathrm{s}}$ is the surface diffusivity, $\quad{ }_{\mathrm{s}}$ is the surface layer width, is the atomic volume, $k_{\mathrm{B}}$ is the Boltzmann's constant and $T$ is the absolute temperature, $\nabla_{\mathrm{s}}^{2}$ is the surface Laplacian (Laplace operator on the surface), and $\quad$ is the curvature of the particle surface.

By solving Eq. (8) and Eq. (9) simultaneously, the co-evolution of particles and grain boundaries in a polycrystalline solid can be tracked. In order to solve these equations, boundary conditions at the particle tip must be considered. The appropriate boundary conditions are the continuity of the chemical potential and surface flux. From Eq. (8) and Eq. (9), this translates into the continuity of ${ }_{\mathrm{s}}$ and $\nabla{ }_{\mathrm{s}}$ at the particle tip. Moreover, mechanical equilibrium requires that the surface tension forces to be balanced at the particle tip. This means that the equilibrium dihedral angle, defined by $=2 \cos ^{1}\left({ }_{\mathrm{b}} / 2_{\mathrm{s}}\right)$ must be maintained during the evolution.

Solving the sharp-interface model for general particle and boundary shapes is extremely difficult [813]. In fact in most cases only 2D solutions for idealized geometries were obtained. Moreover, the boundary separation event is challenging to capture in such models. In [8, 9], only steady-state solutions were sought and boundary separation is assumed to occur when such solutions cannot be found. Other authors [10-12] derived different forms of separation conditions and used them as a priori to simulate the breakaway process. All theses complications have motivated us to employ a phase field (diffuseinterface) description of the problem. Phase field models can easily handle general particle and boundary 8 
morphologies since tracking the interface is not required in these models. Furthermore, boundary separation takes place naturally and no a priori assumptions are required.

\section{Phase field model of particle-grain boundary interactions}

\subsection{Model description}

While existing phase field models study the effect of the presence of immobile particles on grain growth [15-17], a phase field model that investigates the interactions between mobile particles and a migrating boundary was lacking. An early attempt by Vedantam and Mallick [23] was conducted to formulate such model. However, in that model, the particles were assumed to move via a prescribed velocity obtained from the phenomenological models discussed before. In other words, the matter transport mechanism by which the particle migrates was not directly incorporated in that model. The current authors constructed the first phase field models that accounts for the presence of mobile particles on the grain growth kinetics [18-21]. The model can be summarized as follows. A combination of conserved and non-conserved order parameters (phase fields) is used to fully represent the microstructure of a polycrystalline solid containing second-phase particles. The conserved field, $c(x, t)$, represents the solute site fraction, and it takes the value of 1 inside the particle and 0 in the matrix. For the case of pores or bubbles, it represents the site fraction of vacancies or gas atoms. In order to distinguish between different grains with different orientations in the matrix, a set of non-conserved order parameters, , is

used, where $\quad=1,2, \ldots p$, with $p$ being the total number of grains with different orientations. $\quad=1$ in the -th grain and 0 otherwise.

The free energy of the heterogeneous system of particles and grains can be constructed as follows [18-20] 


$$
F=\int f\left(c,{ }_{1}, \ldots, \quad p\right)+\frac{1}{2} \quad|\nabla c|^{2}+\left.\frac{1}{2} \sum_{=1}^{p}|\nabla|\right|^{2} \quad d^{3} r
$$

In the integrand above, the first term represents the bulk free energy density. The two gradient terms account for the excess free energy due to particle (free) surfaces and grain boundaries, respectively. The bulk free energy density used here is a positive-definite multi-well potential that represents the equilibrium phases. It has the form,

$$
\left.f\left(c,{ }_{1}, \ldots, \quad p\right)=A c^{2}\left(\begin{array}{ll}
1 & c
\end{array}\right)^{2}+B\left[\begin{array}{lll}
1 & c
\end{array}\right)^{2}+6 c \sum^{2} 4(1+c) \sum^{3}+3\left(\sum^{2}\right)^{2}\right] .
$$

This particular from has $(p+1)$ minima that correspond to the particle phase and all grains in the solid matrix phase. $A, B, \quad c$ and are material constants related to surface and grain boundary energies.

The conserved density field evolves according to a Cahn-Hilliard equation [24] in the form,

$$
\begin{aligned}
\frac{\partial c}{\partial t} & =\nabla \cdot M \nabla, \\
& =\partial f\left(c,{ }_{1}, \ldots ., \quad, \ldots .,{ }_{p}\right) / \partial c \quad{ }_{c} \nabla^{2} c .
\end{aligned}
$$

Here, $M$ is the mobility and is the chemical potential. Similar to previous studies, we assume that surface diffusion is the dominant mechanism for particle migration. This also allows us to directly compare our results with the predictions of the classical and sharp-interface models discussed above. Nonetheless, other matter transport mechanisms can be accounted for in the model in a straightforward manner. In order to simulate surface diffusion in the phase field approach, a few forms for the mobility were proposed [25-27]. In most cases, the authors demonstrated using a formal asymptotic analysis that their formulation recovers the sharp-interface description of motion by surface diffusion (Eq. (9)). The most common property in all these forms was that the mobility and its first derivative vanish in the bulk phases. Here, we use the following form for the mobility 


$$
M=630 M_{0} c^{4}(1 \quad c)^{4}
$$

where $M_{0}$ (in units of $\mathrm{m}^{5} / \mathrm{Js}$ ) is a material property directly related to the surface diffusivity [20]. The

numerical prefactor in Eq. (13) is chosen such that ${ }^{1} M d c=M_{0}$.

The non-conserved order parameters are governed by Allen-Cahn equations [28] as

$$
\frac{\partial}{\partial t}=L F / \quad=L\left[\partial f\left(c,{ }_{1}, \ldots ., \quad, \ldots .,{ }_{p}\right) / \partial \quad \nabla^{2}\right] \quad, \quad=1,2 \ldots . p .
$$

Here, $L$, the Allen-Cahn mobility, is a material property that is related to the grain boundary mobility [20]. Using constant gradient and mobility coefficients is equivalent to the assumption of isotropic grain boundary energy and mobility.

The phase field model parameters are directly related to the thermodynamic and kinetic parameters that appear in the sharp-interface models (Eqs. 8 and 9). Such relations were established using a formal asymptotic analysis in [20]. This analysis demonstrates that the phase field model recovers the sharpinterface counterpart. From that analysis the model parameters are determined as

$$
\begin{aligned}
& A=\frac{12_{\mathrm{s}} 7 \mathrm{~b}}{\ell}, \\
& B=\frac{\mathrm{b}}{\ell}, \\
&=\frac{3}{4} \mathrm{~b}^{\ell}, \\
& c=\frac{3}{4} \ell\left(2{ }_{\mathrm{s}} \mathrm{b}\right), \\
& L={ }_{\mathrm{b}} M_{b}, \\
& M_{0}\left({ }_{c}+\quad\right)=\frac{\mathrm{s}_{\mathrm{s} \mathrm{s}}}{k_{B} T} .
\end{aligned}
$$


In the above, $l$ is the diffuse interface width. These relations fix all the model parameters in terms of physical material properties, which makes it possible to obtain quantitative results that can be compared with theory and experiments. While the main goal of the current model is to study the effect of mobile particles on the kinetics of grain boundary migration, the model can also be used to investigate the effect of immobile particles by taking their mobility to be zero. Moreover, the model can also be utilized to simulate ideal (particle-free) grain growth if the solute concentration is taken to be zero everywhere. We will take advantage of such generality here to study the particle-grain boundary interactions in detail and quantify their effect on the overall kinetics.

\subsection{Model implementation in MARMOT}

A fully-coupled, fully-implicit finite-element scheme was used to solve the phase field kinetic equations. This scheme was implemented in the phase field code MARMOT [22]. First, the variational (weak) form of the partial differential equations (PDEs) (see Eqs (12) and (14)) was obtained in the regular manner. Specifically, the residual equations in the weak form are

$$
\begin{aligned}
& (,)(\partial f / \partial c,) \quad\left({ }_{c} \nabla c, \nabla\right)+<{ }_{c} \nabla c \cdot \mathbf{n},>=0, \\
& (\partial c / \partial t,)+(M \nabla, \nabla)<M \nabla \cdot \mathbf{n},>=0, \\
& (\partial \quad / \partial t,)+L(\partial f / \partial \quad,)+L(\nabla \quad, \nabla) L<\nabla \quad \cdot \mathbf{n},>=0 \quad, \quad=1,2 \ldots p .
\end{aligned}
$$

In the above is a test function, ( $\Varangle>$ stands for interior integration, and $\langle\Varangle,>$ for boundary integration. Therefore, in this scheme, the fourth-order Cahn-Hilliard equation is transformed into two coupled second-order equations (e.g., Eqs (12a) and (12b)). Solving the Cahn-Hilliard equation in this split form was shown to be a good compromise between accuracy and efficiency [29].

Linear Lagrange discretization of Eqs (16a-16c) employing four-node quadrilateral elements in 2D and eight-node hexahedral elements in 3D was performed. The time integration was carried out via a second-order Backward Differentiation Formula (BDF2). The nonlinear system was solved using the 
preconditioned Jacobian-Free Newton Krylov (JFNK) method [30]. In all simulations, the interface was resolved by at least five elements. Mesh and time step adaptivity were utilized to reduce the computational time. An adaptive mesh will usually be fine in the interfacial regions and coarse in the bulk regions. This is captured in Fig. 1 that depicts a polycrystalline structure with second-phase particles. The figure also demonstrates the fact that the equilibrium particle configuration differs for particle on two-, three-, and four-grain junctions. This stems from the mechanical equilibrium condition, which demands the establishment of the equilibrium dihedral angle at the particle tips. The typical computing time ranges from a few hours to a few days for 2D simulations carried out using 24 cores and for 3D simulations on 216 cores.
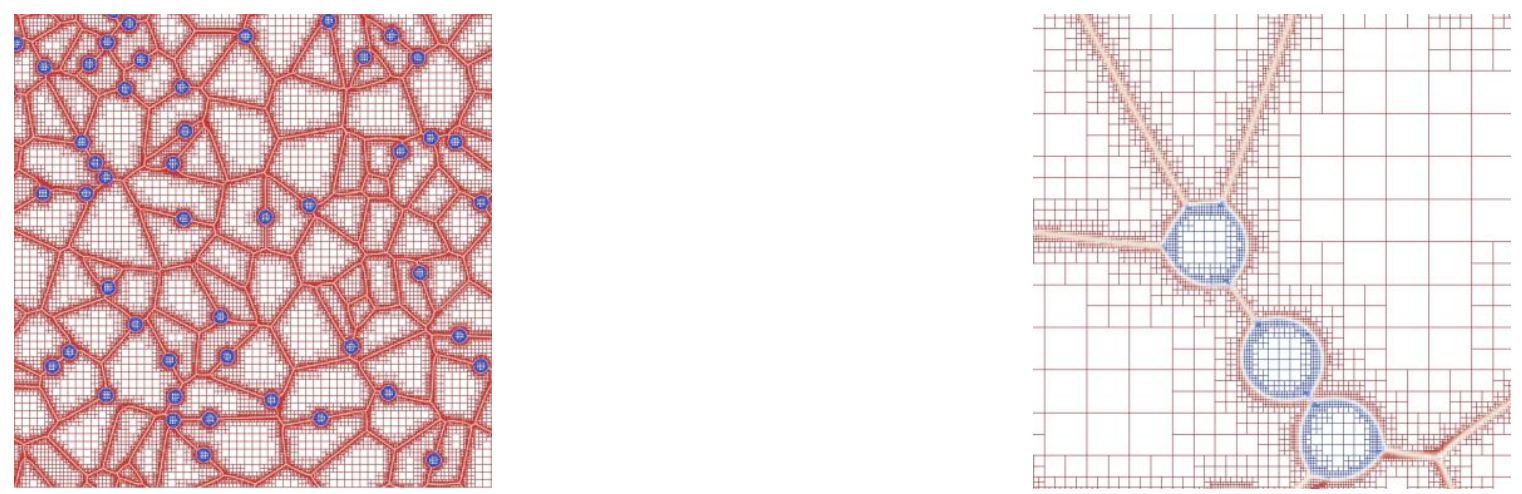

(a) a polycrystal structure with second-phase particles

(b) a close-up view

Fig. 1. An adaptive mesh generated in MARMOT to simulate particle-grain boundary interactions while reducing the computational cost, i.e., the mesh near the interfaces is fine while it is coarse in the bulk regions. Note the different equilibrium particle configurations at two-, three-, and four-grain junctions. In general, the equilibrium particle configuration depends on the dihedral angle and the particle coordination number (e.g., the number of grains attached to the particle).

\section{Results and discussion}

The main goal of the study presented here is to investigate the different particle-grain boundary interactions and their effect on the grain growth kinetics. Therefore, without loss of generality, we use the 
following normalized model parameters: $A=4.25, B=0.25, \quad=3.0,{ }_{c}=9.0, L=1.0$. These parameters give a normalized grain boundary energy, e.g., $\quad{ }_{b}=1.0$, a normalized surface energy, $\mathrm{s}=2.0$, and a normalized interface width, $\ell=4.0$. The particle radius and surface mobility $\left(M_{0}\right)$ were varied to study their influence on the particle-grain boundary interactions. Nonetheless, quantitative results for a specific material of interest can be obtained by using Eq. (15) to relate the model parameters to the thermodynamic and kinetic parameters as we have done before [18-20]. Moreover, to facilitate comparison with classical and sharp-interface models, a few idealized 2D and 3D particle and grain configurations are considered here. Furthermore, for the sake of simplifying the grain growth calculations, particle coarsening (coalescence) is ignored here. To eliminate coalescence in our simulations, we start with a grain size that is much larger than the particle size and stop the simulation before particles start touching. A discussion on how particle coarsening affects the overall grain growth kinetics is presented at the end of this section. Lastly, the function $={ }_{i=1}^{i=p}{ }_{i}^{2}$ was used here to plot all 2D and 3D snapshots; this function takes on the value of 1 inside each grain and 0 inside the particles and changes smoothly across the interfaces.

\subsection{Shrinkage of an isolated circular grain with boundary particles}

Our first case study is the shrinkage of an isolated circular grain embedded in a large matrix grain. The domain size was 912912 and the initial circular grain radius was 300. Periodic boundary conditions were applied in both directions. The particle radius and surface mobility were varied to investigate all possible particle-boundary interactions.

For the case of particle-free grain shrinkage, the boundary velocity is inversely proportional to its radius as given by Eq. (8). This gives rise to the well-known parabolic law, which has the form [1]:

$$
R^{2}(t) \quad R^{2}(0)=k t
$$




$$
k=2{ }_{\mathrm{b}} M_{\mathrm{b}}=2 L
$$

Here, $R(t)$ is the grain radius at time $t, R(0)$ is the initial grain radius, and $k$ is the rate constant. Eq. (15.e) was used to express the rate constant in terms of the phase field model parameters.

On the other hand, in the presence of particles, the boundary velocity is given by Eq. (7) as discussed before. For the current 2D (cylindrical) particle and boundary shapes, $F_{\mathrm{b}}={ }_{\mathrm{b}} / R, N_{\mathrm{p}}=n_{\mathrm{p}} / 2 R L_{\mathrm{cyl}}$, where $n_{\mathrm{p}}$ is the number of particles on the boundary and $L_{\text {cyl }}$ is the length of the cylindrical grain. Moreover, if one ignores coarsening and densification and assume the particle moves along with the boundary as a rigid-body, the particle radius $r$ remains constant and the particle mobility can be derived as $[1-5,8-13]$

$$
M_{\mathrm{p}}=\frac{D_{\mathrm{s} s}}{k_{B} \operatorname{Tr}^{3} L_{\mathrm{cyl}}}
$$

Hence, if one assumes $v=d R / d t$, Eq. (7) can be directly integrated and the final form is

$$
\frac{n_{\mathrm{p}} M_{\mathrm{b}}}{2 L_{\mathrm{cyl}}}[R(t) \quad R(0)]+\frac{M_{\mathrm{p}}}{2}\left[R^{2}(t) \quad R^{2}(0)\right]=\quad{ }_{\mathrm{b}} M_{\mathrm{b}} M_{\mathrm{p}} t .
$$

Clearly, two limiting cases may arise. For the boundary-controlled shrinkage $\left(M_{\mathrm{p}} \square n_{\mathrm{p}} M_{\mathrm{b}} / L_{\text {cyl }}\right)$, the shrinkage kinetics follows Eq. (17) as in the particle-free case. On the other hand, for the particlecontrolled kinetics $\left(M_{\mathrm{p}} \square n_{\mathrm{p}} M_{\mathrm{b}} / L_{\text {cyl }}\right)$, the shrinkage kinetics follows

$$
\begin{aligned}
& R(t) \quad R(0)=k t, \\
& k=2 \quad{ }_{\mathrm{b}} M_{\mathrm{p}} L_{\mathrm{cyl}} / n_{\mathrm{p}}=\frac{2 M_{0}\left({ }_{\mathrm{b}} /{ }_{\mathrm{s}}\right)\left(+{ }_{c}\right)}{n_{\mathrm{p}} r^{3}} .
\end{aligned}
$$

In the second equation, we used Eq. (18) and (15f) to arrive at the second equality that expresses the rate constant in terms of the phase field model parameters. 
All the scenarios discussed above were investigated in our simulations. For the particle-free case, the kinetics followed Eq. (17). The rate constant was calculated to be 5.9, which is very close to the exact value of 6.0 expected from Eq. (17.b). In order to account for the effect of particle drag on the kinetics, 8 particles were evenly distributed on the boundary as shown in Fig. 2. The quasi-rigid-body motion of the particles with the boundary, which is usually assumed in the theoretical models, is evident from the figure. The effect of the surface diffusivity on the kinetics is captured in Fig. 3. The shrinkage kinetics changes from boundary-controlled to particle-controlled as the surface mobility (diffusivity) decreases. For very high surface mobility (see Fig. 3(a)), the particles have almost no effect on the kinetics that was found to follow the parabolic law of Eq. (17) as in the particle-free case. On the other hand, for low surface mobility, the kinetics is particle-controlled and follows the linear law of Eq. (20) as shown in Fig. 3(b). The rate constant for $M_{0}=4.0$ and $r=20$ was found to be $k=510^{4}$ which is relatively close to the exact value $k=7.510^{4}$ calculated from Eq. (20b). For the same particle radius, $k$ also increases linearly with $M_{0}$, in agreement with Eq. (20b), as captured in Fig 3(b). The effect of the particle radius on the particle-controlled kinetics was also studied. Fig. 4 shows the strong dependence of the rate constant on the particle radius for the same surface mobility. The dependence agrees wells with Eq. (20b) as captured in Fig. 4.

We then investigate the effect of the spatial arrangement of the particles on the boundary on the particle-grain boundary interactions. Such effect cannot be captured using the classical models that assume homogeneous microstructure [1, 5-7]. Moreover, most sharp-interface models [8-13] also ignored that effect due to the numerical difficulty with applying the boundary conditions at each particle tip. Here, we simply study this effect by changing the number of particles on the boundary while keeping the particle coverage area (perimeter in 2D) constant. The coverage area is the ratio of the particle surface area to the grain boundary area. We conducted two different simulations. In the first simulation, four particles with a radius of 40 were evenly distributed on the boundary as shown in Fig. 5. The particles 16 
move along with the boundary as in the case for the eight particles discussed above. However, the initially circular grain changes into a near quadrilateral shape. The effect on the kinetics is captured in Fig. 6 that compares the shrinkage rate of the two different configurations with the same coverage and surface mobility (e.g., $M_{0}=4.0$ ). The particle-controlled shrinkage rate for the case of four large particles was four times lower than the case of eight small particles. This is in perfect agreement with the prediction of Eq. (20b) since we doubled the particles radius and decreased their number by one-half. Therefore, for the same particle coverage a few large particles retard the boundary more than many small particles. Hartland et. al. arrived at the same conclusion using their quasi-rigid-body model [14]. In the second simulation only two particles were distributed on the grain boundary as shown in Fig 7. The radius of each particle was 80 so that the coverage is the same as the previous cases. However, in this example the boundary was able to separate from the particles. This can be attributed to the fact that when there are less pining centers (particles) the boundary can easily wrap around the particles and decrease its size till it can reach the critical driving force/velocity required to detach from the particles. Note that Riedel and Svoboda [10, 11] argued that a grain boundary never separates from a 2D (cylindrical) particle, while Petrishcheva and Renner predicted that breakaway could take place in 2D [12]. Our simulations here show that grain boundary separation is possible in $2 \mathrm{D}$, though more suppressed than the $3 \mathrm{D}$ case as will be shown later.

Our last case study for this configuration is devoted to investigate the effect of immobile particles on the grain boundary motion. As we mentioned before, immobile particles are simulated by taking their mobility to be zero (e.g., $M_{0}=0.0$ ). We carried out three different simulations with the same particle coverage and different number and size of particles as for the mobile particle cases discussed above. In all cases a limiting grain size developed and the grain growth process stopped in agreement with the classical Zener model. The establishment of a limiting grain size is demonstrated in Fig 8 that shows the evolution of the shrinking grain size. Note that the limiting grain size is defined for the growing grain, so a larger final size of the shrinking grain means a smaller limiting grain size. As evident from the figure, the 
limiting grain size decreases (the final size of the shrinking grain increases) as the number of particles increases (and hence their size decreases). Therefore, for immobile particles, many small particles pin the boundary more effectively than a few large particles. Note this is opposite to the case of mobile particles as we discussed above.
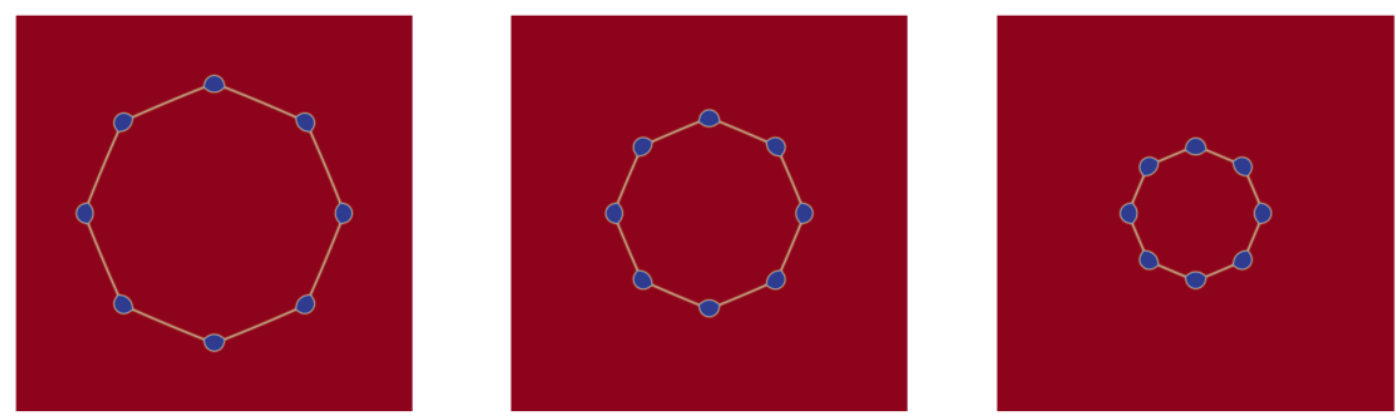

Fig. 2. Snapshots of the shrinkage of an isolated circular grain with boundary particles. The particles take on their equilibrium lenticular shape. For high enough surface mobility, the particles move along with the boundary as a rigid-body.

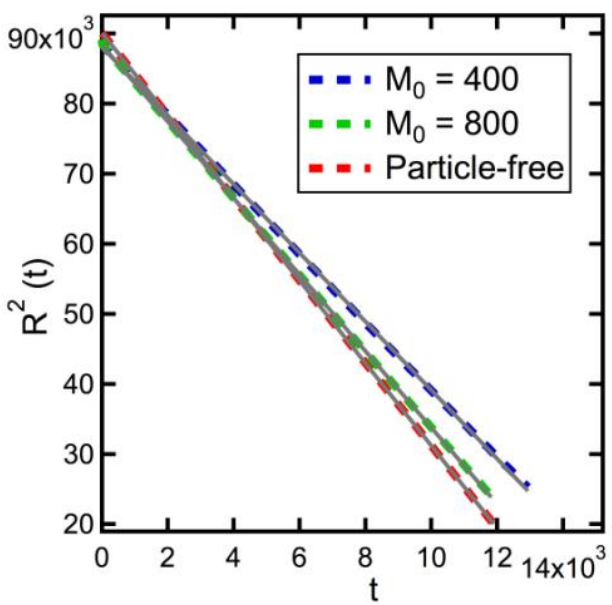

(a) High surface mobility

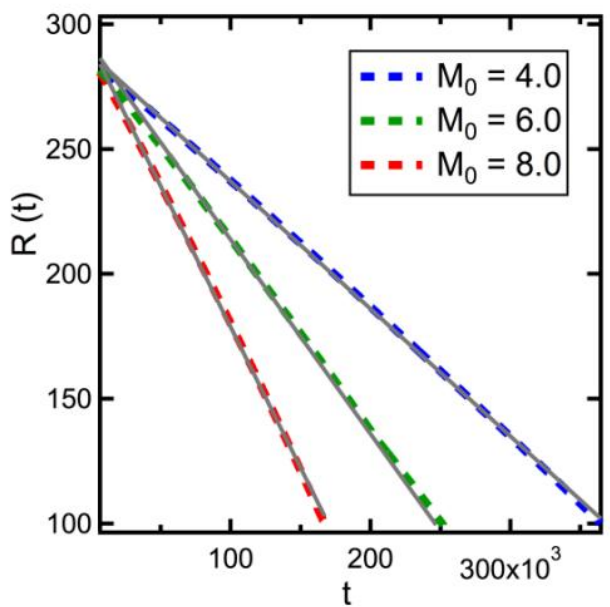

(b) Low surface mobility

Fig. 3. The dependence of the shrinkage kinetics on the surface mobility for the configuration shown in Fig. 2. The particle radius was 20. (a) For high surface mobility, the particles almost exert no drag on the boundary and the kinetics follows the parabolic law given by Eq. (17) as in the particle-free case, (b) for 
low surface mobility, the boundary motion is dictated by the particle kinetics. The particle-controlled kinetics follows the linear law in Eq. (20). For the particle-controlled kinetics, the rate constant increases linearly with the surface mobility in agreement with Eq. (20b). The dotted lines are the simulations results and the solid lines are the best fittings.
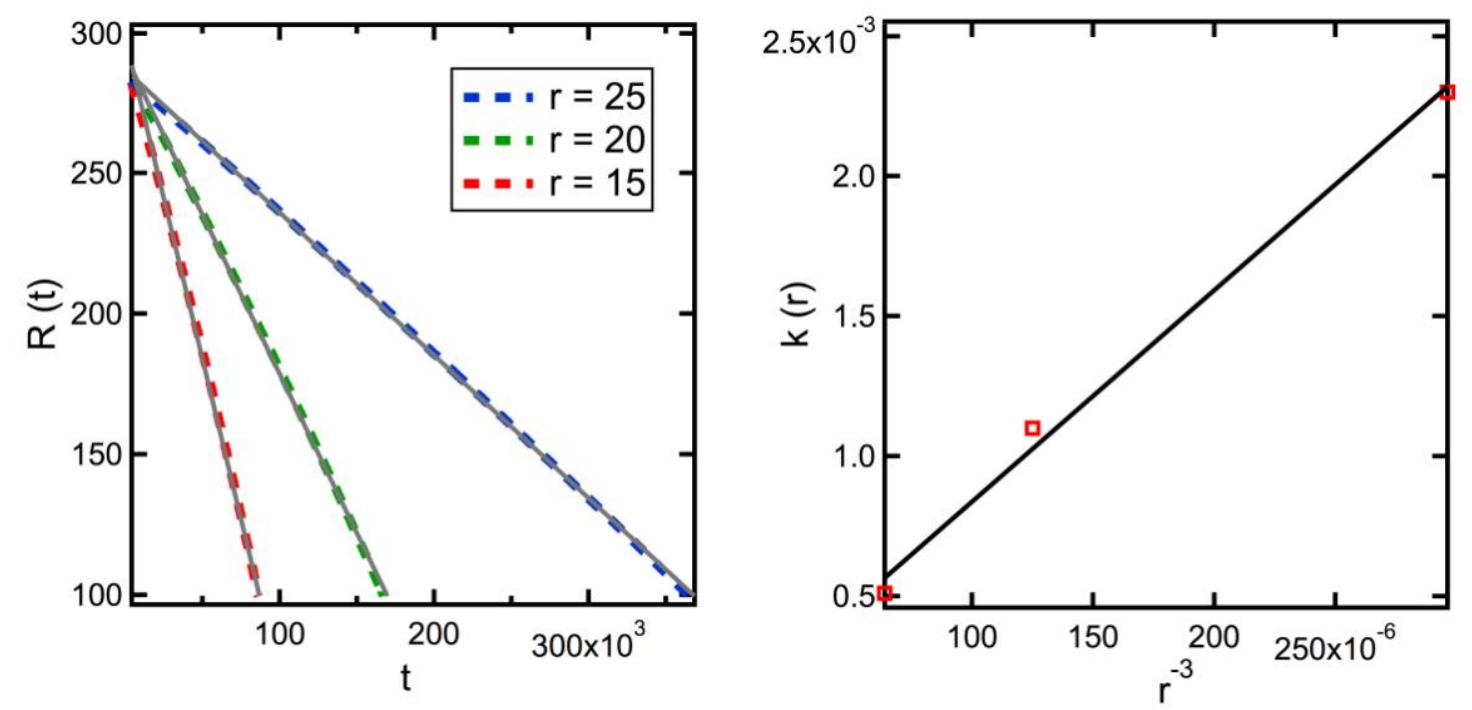

Fig. 4. The dependence of the particle-controlled shrinkage kinetics on the particle radius. The evolution of the grain radius as function of the particle radius is shown in the left figure. The dependence of the rate constant on the particle radius agrees well with Eq. (20b) as captured in the figure on the right. The dotted lines are the simulations results and the solid lines are the best fittings.
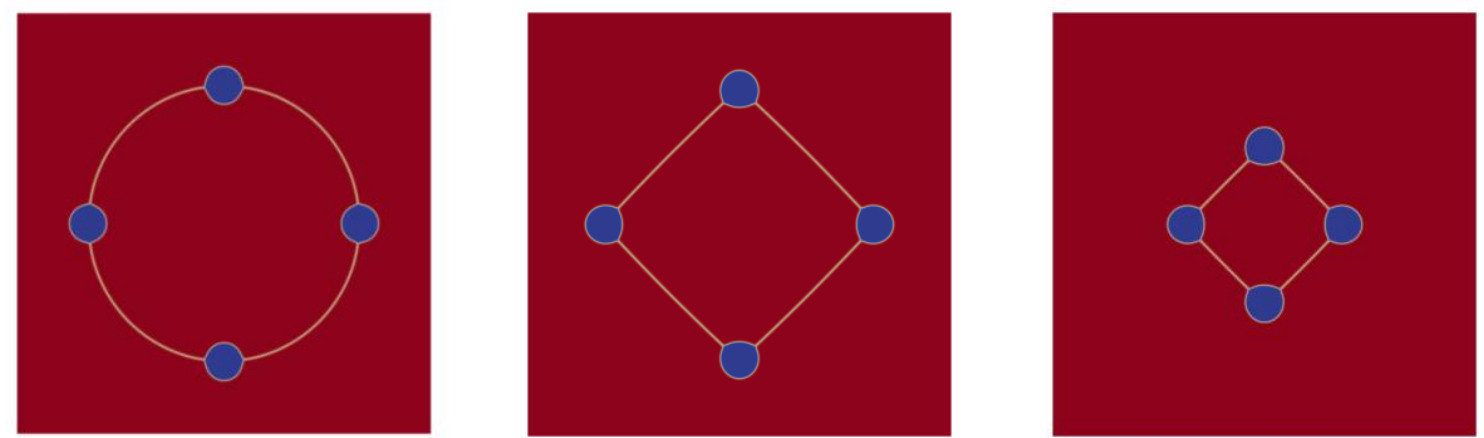
Fig. 5. Snapshots of the shrinkage of an isolated circular grain with four boundary particles with the same coverage as for the eight particles case presented in Fig. 2. The particles move along with the boundary as a rigid-body as in Fig. 2, but the shrinkage is more anisotropic here, i.e., the initially circular grain transform into a near quadrilateral shape.

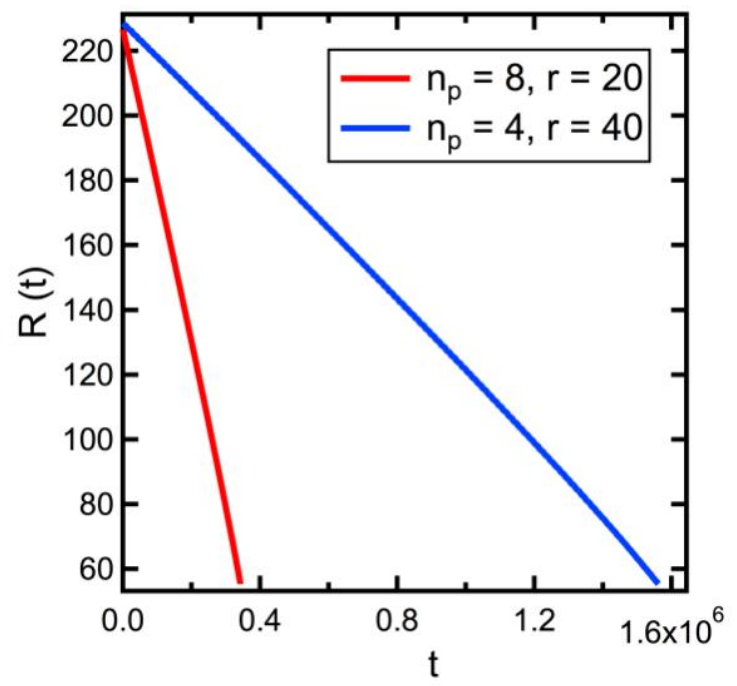

Fig. 6. Comparison of the shrinkage kinetics of a circular grain with different number and size of boundary particles and same particle coverage and surface mobility (see Figs. 2 and 5). In both cases, the shrinkage process is particle-controlled and follows Eq. (20). The rate constant for the case of small particles was four times larger than the rate constant for the case of large particles.
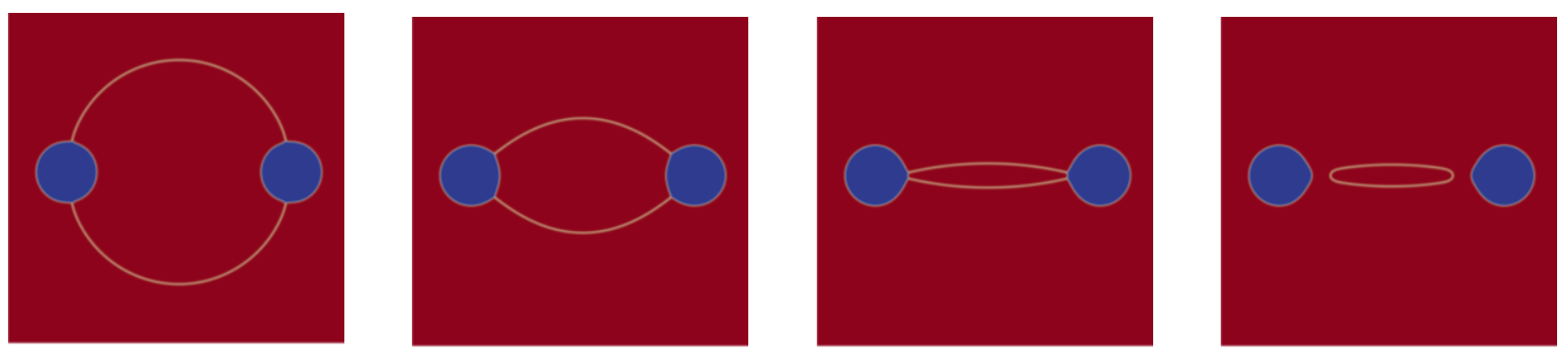

Fig. 7. Snapshots of the shrinkage of an isolated circular grain with two boundary particles with the same coverage as for the cases presented in Fig. 2 and Fig. 5. In this configuration, however, the grain boundary detaches from the particles. In this particular case, with less number of particles, the grain 20 
boundary was able to wrap around the particles and decrease its size to the critical value required to break away from the particles (recall that the intrinsic grain boundary velocity is inversely proportional to its size).

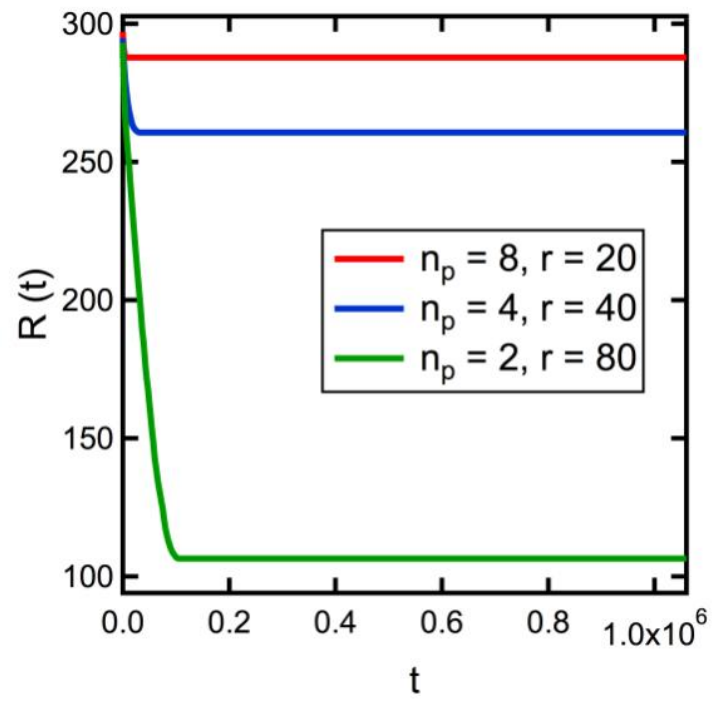

Fig. 8. Effect of the presence of immobile particles on the shrinkage kinetics of a circular grain. In contrast to mobile particles, immobile particles (e.g., with zero mobility) completely pin the grain boundary and stop the grain growth/shrinkage process.

\subsection{Shrinkage of a four-sided grain with edge or corner particles}

According to the well-known topological analysis of grain growth put forward by Neumann and Mullins [1,31], a grain with more than six sides will grow, while a grain with less than six sides will shrink. A grain with six sides is static. The growth/shrinkage rate of a grain with $n$ sides is given by

$$
\frac{d A(n)}{d t}=\frac{-}{3} \quad{ }_{\mathrm{b}} M_{\mathrm{b}}\left(\begin{array}{ll}
n & 6
\end{array}\right)
$$

where, $A(n)$ is the area of a grain with $n$ sides. Klinger et. al. have generalized the analysis to the case where mobile particles (pores) are present on the grain boundary [13]. They have derived an equation for the grain size that has the form 


$$
\frac{d R}{d t}=\frac{\sqrt{3} M_{\mathrm{b}} M_{\mathrm{p}}\left(\begin{array}{ll}
n & 6
\end{array}\right)}{6\left(\sqrt{3} M_{\mathrm{p}} R+n_{\mathrm{p}} M_{\mathrm{b}} / L_{\mathrm{cyl}}\right)}
$$

This form indicates that also a grain with more than six sides grows, while one with less than six sides shrinks as in the particle-free case. However, the growth/shrinkage kinetics is now dependent on the number of particles and their mobility. Again, if one assumes the particle size remains constant during grain growth/shrinkage, Eq. (22) can be directly integrated to give a formula equivalent to Eq. (19), e.g.,

$$
\left.6 \frac{n_{\mathrm{p}} M_{\mathrm{b}}}{L_{\mathrm{cyl}}}[R(t) \quad R(0)]+3 \sqrt{3} \quad M_{\mathrm{p}}\left[R^{2}(t) \quad R^{2}(0)\right]=\sqrt{3} \quad M_{\mathrm{b} \quad{ }_{\mathrm{b}} M_{\mathrm{p}}(n} 6\right) t .
$$

In fact the shrinkage of an isolated circular grain as given by Eq. (19) is a special case of Eq. (23) when the number of grain sides (or neighboring grains), $n$, goes to zero. In other words, one can arrive at the same evolution equation for the grain size using two different approaches, e.g., the effective mobility approach used here and the topological analysis approach utilized in [13].

In the same manner as before, two limiting cases can be identified. For the boundary-controlled shrinkage ( $M_{\mathrm{p}} \square 2 n_{\mathrm{p}} M_{\mathrm{b}} / \sqrt{3} L_{\text {cyl }}$ ), the shrinkage kinetics follows the Neumann and Mullins relation (e.g., Eq. (21)) as in the particle-free case. On the other hand, for the particle-controlled kinetics

$$
\begin{aligned}
& \left(M_{\mathrm{p}} \square 2 n_{\mathrm{p}} M_{\mathrm{b}} / \sqrt{3} L_{\mathrm{cyl}}\right), \text { the growth/shrinkage kinetics follows } \\
& R(t) \quad R(0)=k t \\
& \quad k=\left(\begin{array}{ll}
n & 6
\end{array}\right) \quad{ }_{\mathrm{b}} M_{\mathrm{p}} L_{\mathrm{cyl}} / 2 \sqrt{3} n_{\mathrm{p}}=\frac{\left(\begin{array}{ll}
n & 6
\end{array}\right) M_{0}\left({ }_{\mathrm{b}} /{ }_{\mathrm{s}}\right)\left({ }_{c}\right.}{2 \sqrt{3} n_{\mathrm{p}} r^{3}} .
\end{aligned}
$$

In Eq. (24b), Eqs. (18) and (15f) were used to relate the rate constant to the phase field model parameters.

We study here the shrinkage of a four-sided grain. The initial effective grain radius of the four-sided grain was 200. We define here an effective grain radius as the radius of an effective circle with the same area of the four-sided grain. In this example, natural boundary conditions for all the variables were 
applied. Two different particle configurations were studied, e.g., edge (two-grain junction) particles and corner (triple junction) particles. Snapshots of the shrinkage of a four-sided grain with edge/corner particles are presented in Fig. 9. Similar to the circular grain case, the particles migrate with the boundary as a quasi-rigid-body. The equilibrium shapes of edge and corner particles are kept during the evolution as evident from the figure.

The kinetics of the shrinkage process is captured in Fig. 10. For the particle-free case, the area decreased linearly with time, in agreement with Eq. (21), as shown in Fig. 10(a). The rate constant was found to be 5.9, which is slightly lower than the exact value of 6.28. The effect of edge or corner particles on the particle-controlled kinetics is shown in Fig. 10(b). For the case of edge particles with $M_{0}=4.0$ and $r=20$, the rate constant take on the value $k=3.310^{4}$, which is relatively close to the exact value $k=4.310^{4}$ calculated from Eq. (24b). For corner particles, the rate constant was $k=2.810^{4}$. Therefore, edge particles are more mobile and retard the grain boundary less than corner particles. This prediction is in agreement with the analysis by Petrishcheva and Renner [12]. We also investigated the effect of surface mobility, particle size, and immobile particles on the shrinkage kinetics and the results were similar to those presented before for the circular grain case. Therefore, we do not include these results here to avoid duplication. 

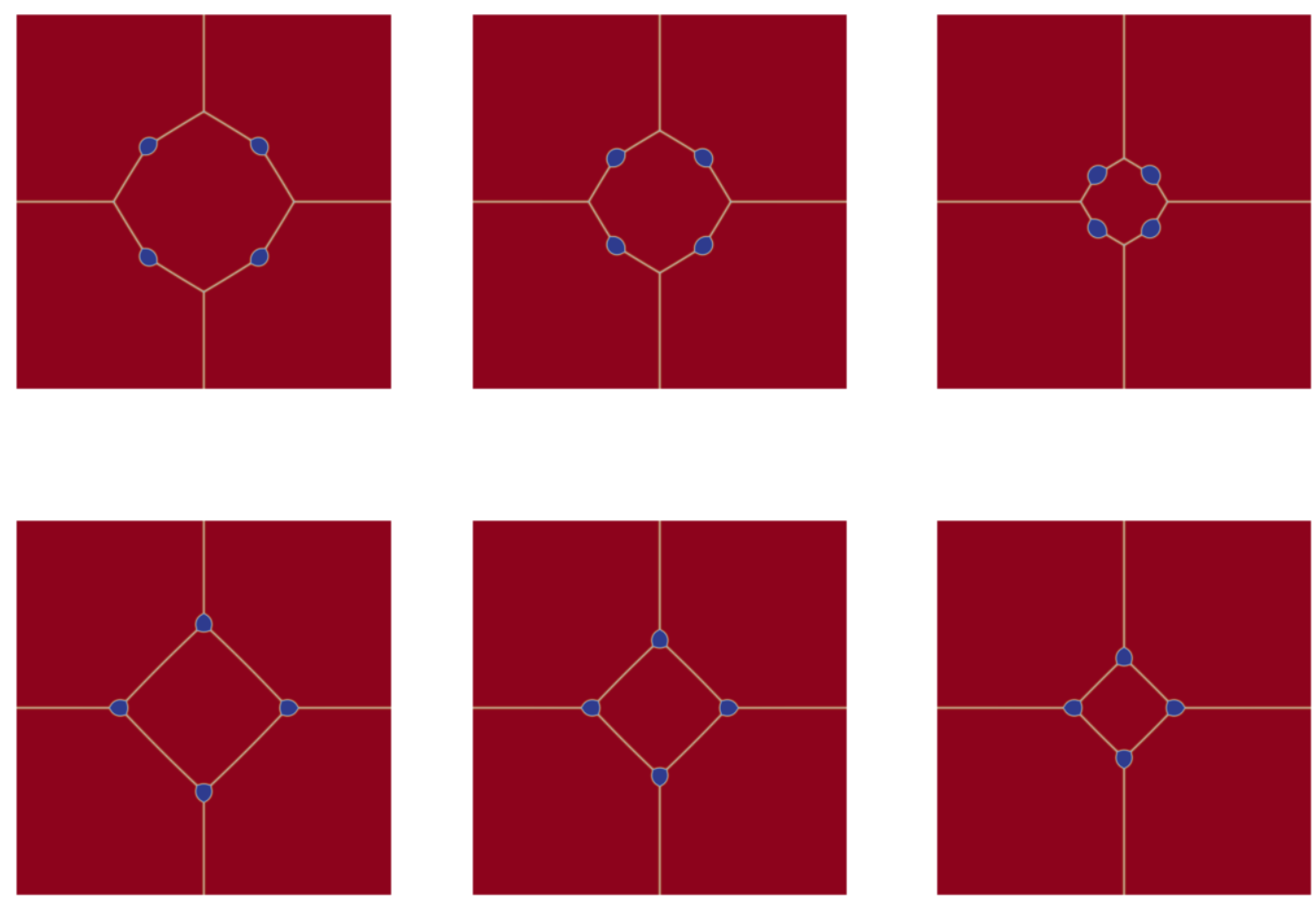

Fig. 9. Snapshots of the shrinkage of a four-sided grain with edge (two-grain junction) particles (upper row) and corner (triple junction) particles (lower row). Similar to the shrinkage of a circular grain, the quasi-rigid-body motion of the particles with the boundary is evident. Note the different equilibrium shapes of edge and corner particles. 


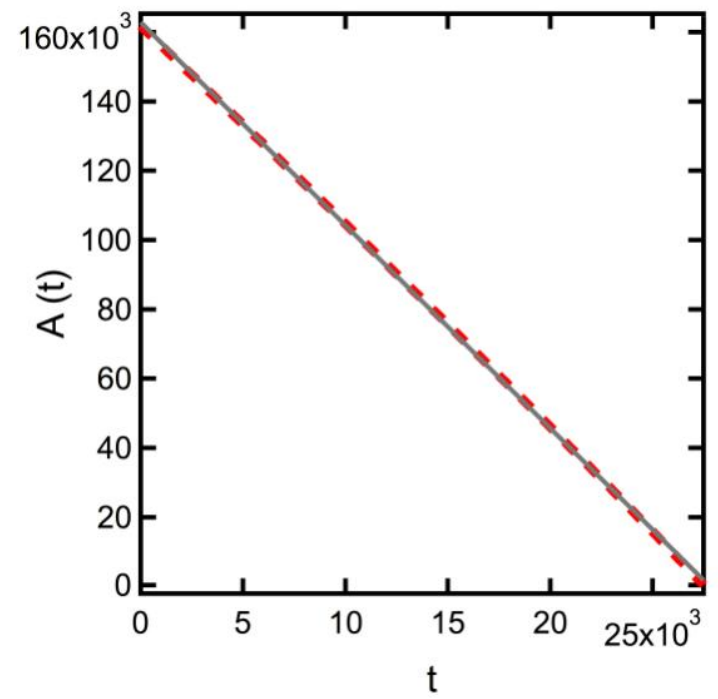

(a) Particle-free shrinkage

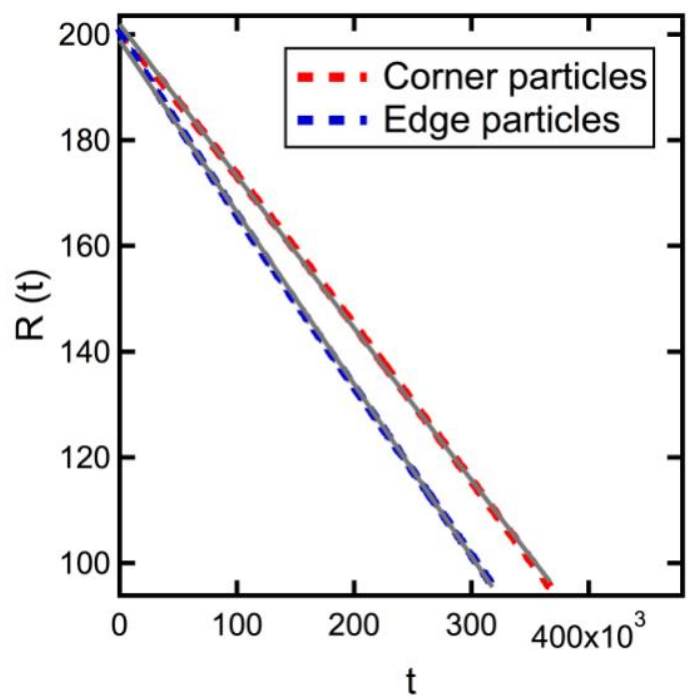

(b) Particle-controlled shrinkage

Fig. 10. Shrinkage kinetics of a four-sided grain, (a) particle-free case, (b) particle-controlled case with edge/corner particles located on the boundary. The kinetics in (a) and (b) follows closely Eq. (21) and Eq. (24), respectively (see text). Corner particles retard the shrinkage/growth kinetics more than edge particles. The dotted lines are the simulations results and the solid lines are the best fittings.

\section{$4.33 D$ simulations of the shrinkage of a cylindrical grain with spherical boundary particles}

We now study the 3D particle-grain boundary interactions. We investigate here the shrinkage of a cylindrical grain embedded in a large matrix grain with spherical particles located on its boundary. The nature of drag and motion of 3D spherical particles is completely different from the 2D cylindrical particles discussed above [1-5, 8-14]. For instance, for the case of immobile particles, the drag force experienced by the boundary depends on the particle shape (recall Eqs. 3 and 4) [16]. For the case of mobile particles, the particle mobility depends on the particle shape [1, 8-12]. Moreover, it is well-known that it is easier for a migrating boundary to break away from a 3D spherical particle than from a $2 \mathrm{D}$ (cylindrical) particle [8-15]. 
Following the same procedure conducted for the shrinking of an isolated circular grain, one can show that the evolution equation for the cylindrical grain radius is the same as Eq. (19). However, the particle mobility of the 3D spherical particle is now given by (to be compared with Eq. (18)) [1, 8-11]

$$
M_{\mathrm{p}}=\frac{D_{\mathrm{s} \mathrm{s}}}{k_{B} T r^{4}}
$$

Again as in the 2D case, for the case of particle-free or boundary controlled kinetics, the shrinkage proceeds according to the parabolic law of Eq. (17). For the case of particle-controlled shrinkage, the grain radius decreases linearly with time as in Eq. (20a), but the rate constant has now the form (compare with Eq. (20b))

$$
k=2 \quad{ }_{\mathrm{b}} M_{\mathrm{p}} L_{\mathrm{cyl}} / n_{\mathrm{p}}=\frac{2 L_{\mathrm{cyl}} M_{0}\left({ }_{\mathrm{b}} /{ }_{\mathrm{s}}\right)\left(+{ }_{c}\right)}{n_{\mathrm{p}} r^{4}} .
$$

We carried out several 3D simulations to study the 3D particle-grain boundary interactions in detail. In our 3D simulations, the domain size was 500500 160. The initial cylindrical grain radius was 150 and its height was 160. Periodic boundary conditions were applied in all directions. As shown in Fig. 11, 16 particles were uniformly distributed on the boundary. Different values for the particle radius and surface mobility were used to study their effects on the kinetics. As clear from Fig. 11, for high enough surface mobility $\left(\begin{array}{ll}M_{0} & 0.5\end{array}\right)$, the particles move along with the boundary as a quasi-rigid-body similar to the $2 \mathrm{D}$ case. The kinetics of the shrinkage of the cylindrical grain is captured in Fig. 12 and Fig. 13. For the particle-free case, the kinetics followed the parabolic law of Eq. (17) as clear from Fig. 12(a). For the particle-controlled case, the kinetics followed Eq. (20a) with the rate constant now given by Eq. (26). The linear dependence of the rate constant on the surface mobility (see Eq. (26)) is captured in Fig. 12(b). The strong dependence of the rate constant on the particle size (e.g., $k \mu r^{4}$ ) is also consistent with Eq. (26) as obvious from Fig. 13. 
As mentioned above, a boundary can separate from 3D spherical particles easier than from 2D cylindrical particles [8-15]. Recall that in our 2D simulations, boundary breakaway occurred only for a particular configuration (e.g., Fig 7) where the grain boundary wrapped around the two particles and attained its critical velocity. While, in contrast to the sharp-interface models [10-12], no a priori assumption about the separation condition must be adopted and implemented numerically, we consult such expression to guide our selection of parameters for the simulations. The separation condition was given by Eq. (6). Note that for the particle-controlled case, the second term on the right hand side of Eq. (6) can be neglected and the separation condition reduces to the same condition for the immobile particle case, e.g., $F_{\mathrm{b}}>N_{\mathrm{p}} F_{\mathrm{p}}$. For the $3 \mathrm{D}$ configuration considered here and assuming the particle exerts the maximum drag force, e.g., $F_{\mathrm{p}}=r_{\mathrm{b}}$ (see Eq. (3)), the breakaway condition simply reduces to $2 L_{\mathrm{cyl}}>n_{\mathrm{p}} r$. We ran multiple 3D simulations with $L_{\mathrm{cyl}}=160, n_{\mathrm{p}}=16, M_{0} \quad 2.0$, and different values for the particles radius for each simulation. Grain boundary separation occurred only when $r<18$, even for the case of immobile particles, which is very close to the prediction from the separation condition, e.g., $r<20$ given the diffuse-interface nature of the particle surface. Snapshots of the separation of the grain boundary from the particles are presented in Fig. 14.

As we demonstrated in the 2D simulations, the spatial arrangement of the particles on the boundary has a prominent effect on the particle-grain boundary interactions and the overall growth/shrinkage rate. Therefore, we also investigate here the effect of the spatial arrangement of the particles on the boundary on the breakaway process. We consider particles with low mobility $\left(\begin{array}{ll}M_{0} & 2.0\end{array}\right)$ and immobile particles. We evenly distributed 10 particles with $r=32$ on only one-half of the grain boundary as shown in Fig. 15. This configuration produces the same total maximum drag force as the configuration of 16 particles with $r=20$, while has higher particle coverage area and volume fraction. Recall that for the configuration of 16 particles with $r=20$ (see Fig. 11 and Fig. 12) the grain boundary could not separate 
from the particles. On the other hand, for the configuration of 10 particles with $r=32$, the boundary was able to detach from the particles as demonstrated in Fig. 15. As also evident from the figure, the boundary does not separate form all the particles at the same time, instead it completes the process on a few steps. Each step can be considered as a partial breakaway event. Therefore, the non-uniform distribution of particles on the boundary facilitates the process of boundary detachment. Note that the classical phenomenological models [1,5-7] that assume homogeneous microstructure cannot predict such effect.
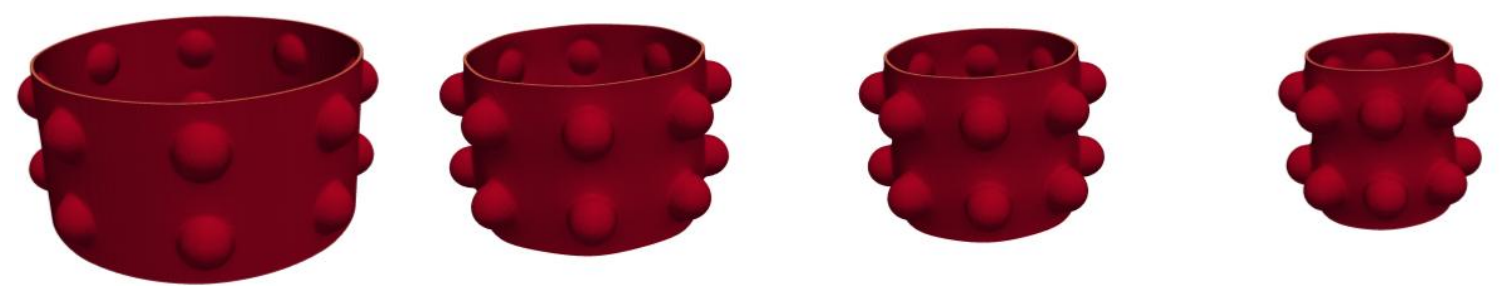

Fig. 11. Snapshots of the shrinkage of a cylindrical grain with spherical boundary particles. For high enough surface mobility $\left(M_{0} \quad 0.5\right)$, the particles move along with the boundary as a quasi-rigid-body. 


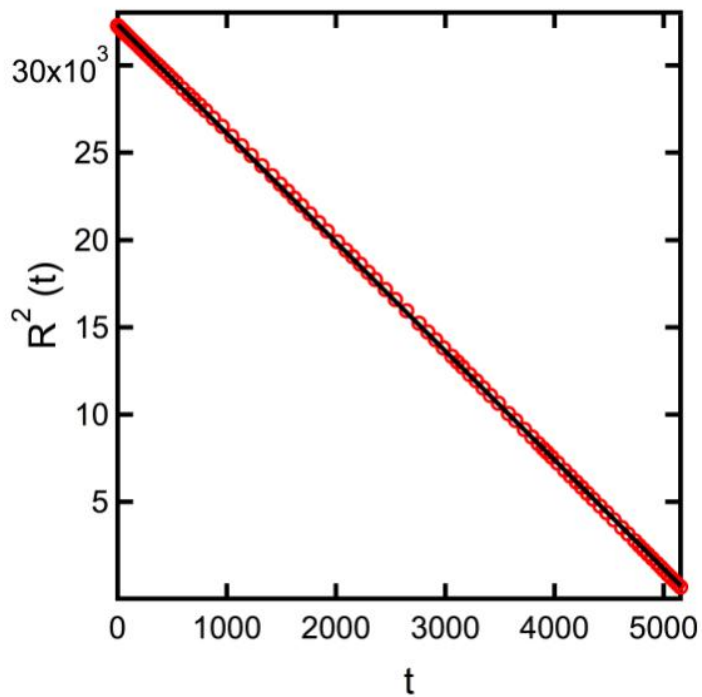

(a) Particle-free kinetics

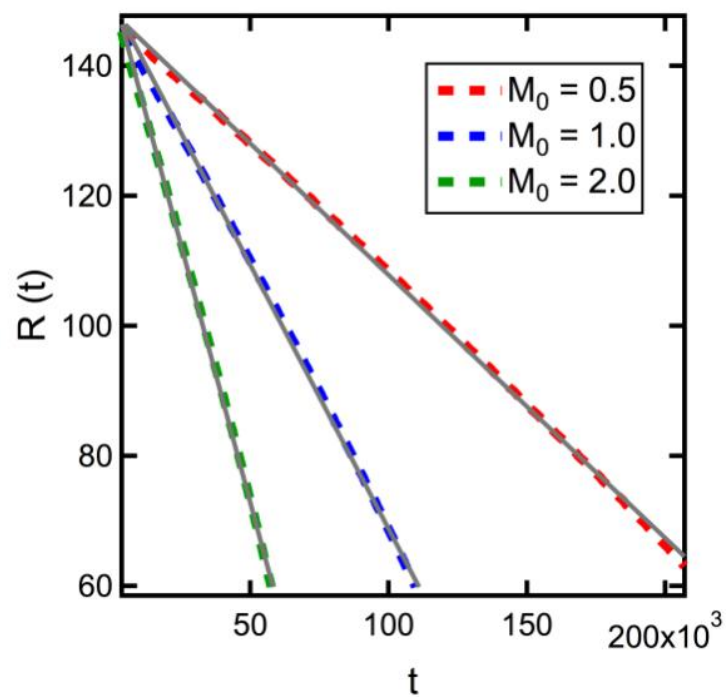

(b) Particle-controlled kinetics

Fig. 12. The shrinkage kinetics of a 3D cylindrical grain with spherical particles on its boundary (see Fig. 11). (a) The particle-free kinetics follows the parabolic law given by Eq. (17), (b) the particle-controlled kinetics follows the linear law of Eq. (20a) with the rate constant given by Eq. (26). For the particlecontrolled kinetics, the rate constant increases linearly with the surface mobility $\left(M_{0}\right)$ in agreement with Eq. (26). The particle radius in (b) was 20. The dotted lines are the simulations results and the solid lines are the best fittings. 

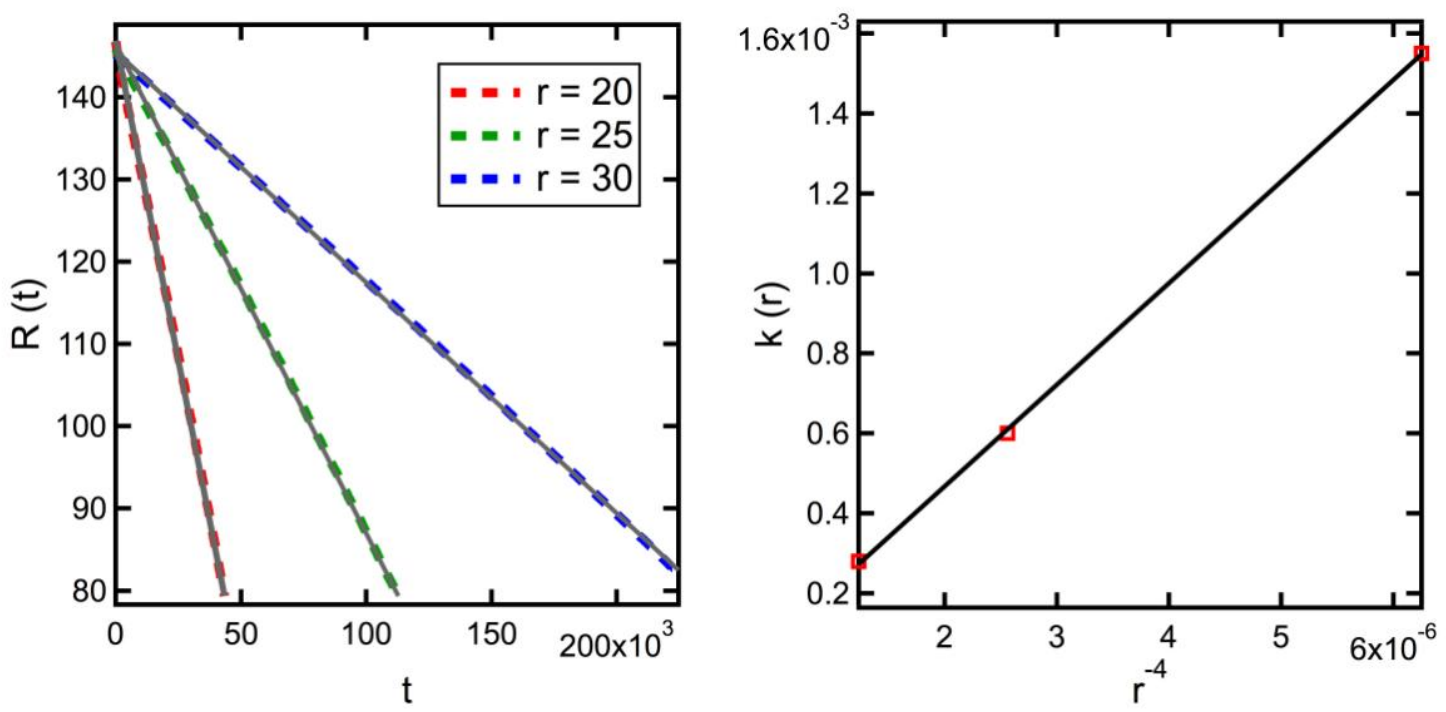

Fig. 13. The dependence of the particle-controlled (here $M_{0}=2.0$ ) shrinkage kinetics on the particle radius for the 3D configuration shown in Fig. 11. The evolution of the grain radius as function of the particle radius is shown in the left figure. The dependence of the rate constant on the particle radius agrees well with Eq. (26) as captured in the figure on the right. The dotted lines are the simulations results and the solid lines are the best fittings.
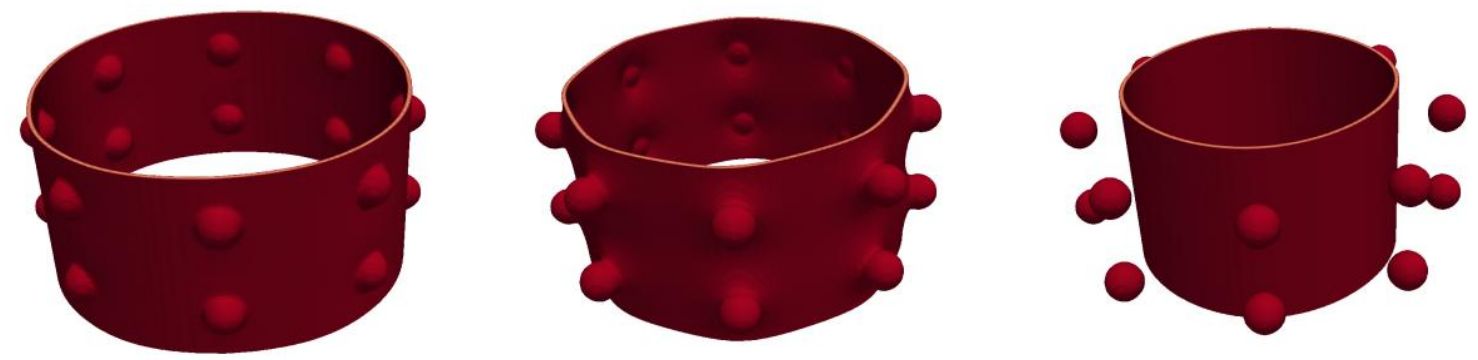

Fig. 14. Snapshots of the separation of a migrating boundary from second-phase particles. Boundary breakaway took place only when the particle radius was smaller than 18 in agreement with the separation condition developed in the text. 

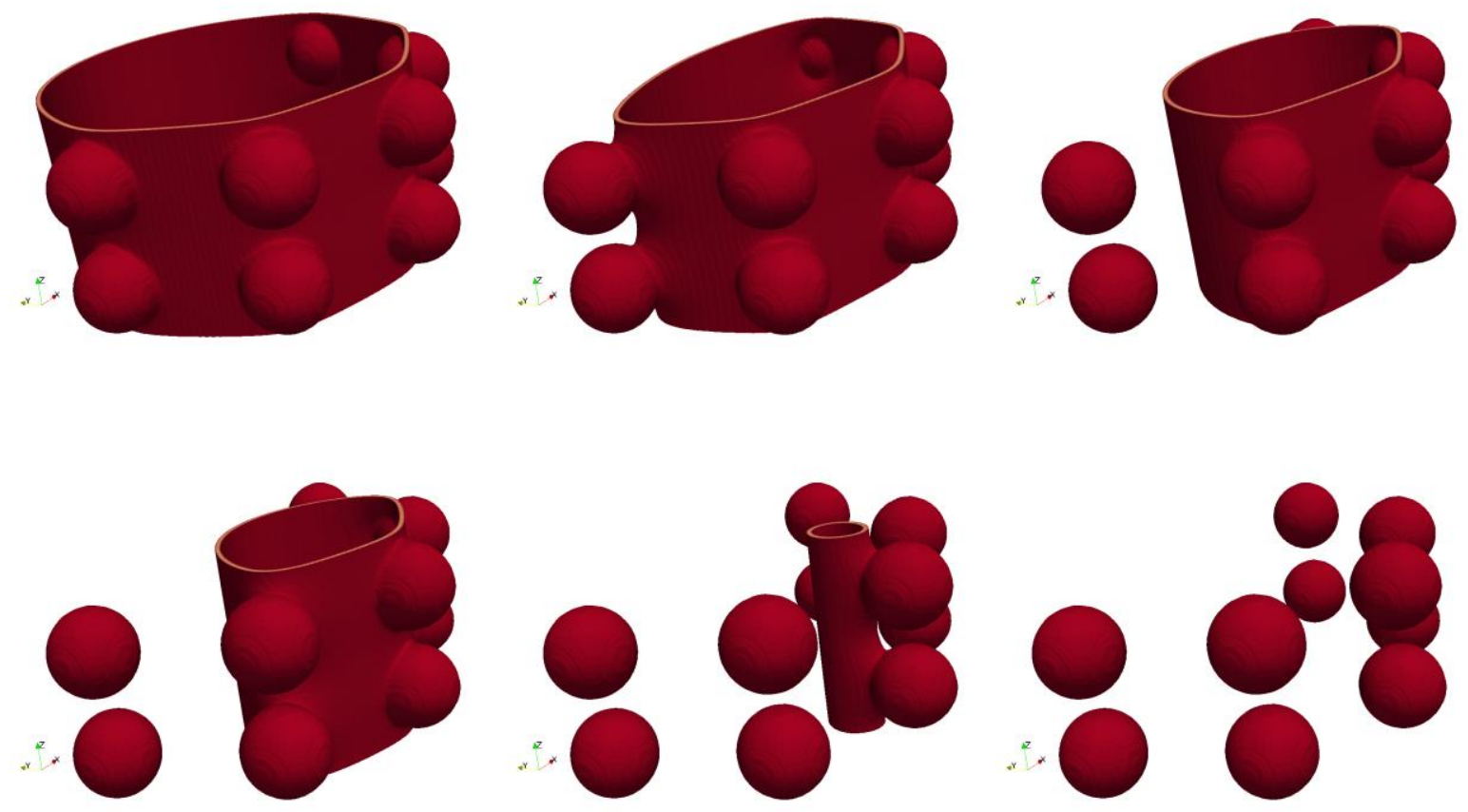

Fig. 15. Snapshots of the separation of a migrating boundary from second-phase particles that are evenly distributed on only one-half of the boundary. Note that the boundary breaks away from the particles successively and not at once (partial breakaway). Therefore, the non-uniform distribution of particles on the boundary increases the possibility of boundary detachment. 


\subsection{Comment on concurrent grain growth, particle coarsening, and densification}

Lastly, we briefly discuss the complicated situation of concurrent grain growth, particle coarsening, and particle shrinkage (densification) that occurs during sintering [1]. In such a case, the particle size changes with time and hence the evolution equations for the grain size derived above (Eq. (19) or Eq. (23)) are no longer valid. Instead, one should solve Eq. (7) simultaneously with an evolution equation for the particle radius. An equation for the change of pore radius due to shrinkage and coarsening during sintering was derived by Klinger et. al. [13]. A simplification can be achieved if densification can be ignored, which is usually the case during the final stage of sintering [1, 8-12]. Assuming the coarsening rate of the particles is proportional to the grain growth rate [1], one expects that $r(t) \mu R(t)$; and hence the integration of Eq. (7) with the particle mobility given by Eq. (18) or Eq. (25) now gives

$$
\frac{n_{\mathrm{p}} M_{\mathrm{b}}}{8(d \quad 1)}\left[R^{4}(t) \quad R^{4}(0)\right]+\frac{D_{\mathrm{s} \mathrm{s}}}{2 k_{B} T}\left[R^{2}(t) \quad R^{2}(0)\right]=\left(\begin{array}{ll}
d & 1
\end{array}\right){ }_{\mathrm{b}} M_{\mathrm{b}} \frac{D_{\mathrm{s} \mathrm{s}}}{k_{B} T} t .
$$

Here $d$ is the dimensionality of the problem, e.g., 2 or 3. Note that Eq. (27) now predicts a growth exponent of 4 for the particle-controlled kinetics as opposed to the expected value of 1 in the absence of particle coarsening (recall Eq. (20) or Eq. (24)). The current phase field model can simulate particle coarsening/coalescence since it takes place via the same mechanism that facilitates particle migration (e.g., surface diffusion). In fact our previous $2 \mathrm{D}$ and 3D simulations of grain growth in porous polycrystalline solids predicted a growth exponent that lies between 2 and 4 in agreement with Eq. (27) [18-20]. Klinger et. al. [13] predicted the same range for growth exponent even when densification was taken into consideration. Riedel and Svoboda also derived similar equations using energy dissipation arguments $[10,11]$. Therefore, we demonstrated here that similar evolution equations for the grain size and growth laws can be obtained using different approaches such as the effective mobility approach of Brook and others (which we followed here) [1, 6], the topological analysis of Klinger et. al. [13], or the energy dissipation principles of Riedel and Svoboda $[10,11]$. It should also be noted that several grain 
growth experiments for different materials reported a growth exponent in the range between 2 and 4 [1, 32, and 33].

\section{Concluding remarks}

A quantitative phase field model was utilized to study particle-grain boundary interactions and their effect on the grain boundary migration. The model couples the curvature-driven motion of grain boundary with particle migration via surface diffusion. The phase field model alleviates all the restrictive assumptions of the classical homogeneous models and obviates all the numerical difficulties of the sharpinterface models. The kinetic equations of the model were solved using a fully-coupled, fully-implicit finite-element scheme implemented in MARMOT.

Our simulations demonstrated that the phase field model captures all possible particle-grain boundary interactions. Immobile particles can completely pin the boundary and stop the grain growth process. For particles with high enough surface mobility, the particles move along with the migrating boundary as a rigid-body. For low surface mobility, the boundary can break away from the particles. When the boundary drags the particles along with it, the overall growth may be controlled by the boundary or the particle kinetics. Evolution equations for the grain size were derived that show the transition from boundary-controlled kinetics to particle-controlled as the particle mobility decreases or the particle size increases. The grain growth exponent lies between 1 and 2 in the absence of particle coarsening. When grain growth and particle coarsening occur simultaneously, the grain growth exponent ranges form 2 to 4 . For the particle-controlled shrinkage/growth, the dependence of the growth/shrinkage rate on the particle mobility and size agrees well with the predictions from theoretical models. It was found that, for a given particle coverage, a few large particles retards the gain boundary migration more than many small particles during particle-controlled grain growth. Moreover, it was demonstrated that the particle morphology also affects the kinetics. Particles on triple junctions retard the grain 
growth/shrinkage more than particles on two-grain junctions. Furthermore, it was shown that a migrating boundary could easily separate from a 3D spherical particle, while it can hardly break away from a 2D cylindrical particle. The non-uniform distribution of particles was found to facilitate boundary breakaway. While, in order to directly compare our model results with their counterparts from the phenomenological and sharp-interface models, only simulations of idealized particle and grain boundary shapes (e.g., cylindrical and spherical) were presented here, the phase field model can be applied to general configurations where theoretical models cannot produce predictive results.

While surface diffusion is considered here to be the dominant mechanism of particle migration, other mechanisms such as bulk diffusion and evaporation and condensation can be incorporated into the model in a straightforward manner. The particle surface and grain boundary energies and mobilities were assumed to be isotropic here. The anisotropy of these parameters can be taken into consideration in phase field models in a systematic way as shown in [34-36]. Particle shrinkage was ignored in this study. However, this process is important for the study of sintering where densification proceeds via pore shrinkage. Densification can be treated in the phase field approach by considering the rigid-body translational and rotational motion of the grains and stresses as was demonstrated in $[37,38]$.

\section{Acknowledgement}

This work was funded by the Department of Energy Nuclear Energy Advanced Modeling and Simulation program. This manuscript has been authored by Battelle Energy Alliance, LLC under Contract No. DEAC07-05ID14517 with the US Department of Energy. The United States Government retains and the publisher, by accepting the article for publication, acknowledges that the United States Government retains a nonexclusive, paid-up, irrevocable, world-wide license to publish or reproduce the published form of this manuscript, or allow others to do so, for United States Government purposes.

\section{References}

[1] M. N. Rahaman, Ceramic Processing and Sintering. New York: Marcel Dekker; 2003. 
[2] D.W. Kingery, H.K. Bowen, D.R. Uhlmann, Introduction to Ceramics. New York: Wiley; 1976.

[3] R.W. Armstrong, Met. Trans. 1 (1970) 1169.

[4] J.A. Turnbull, J. Nucl. Mater. 50 (1974) 62.

[5] F.A. Nichols, J. Am. Ceram. Soc. 51 (1968) 468.

[6] R. Brook, J. Am. Ceram. Soc. 52 (1969) 56.

[7] F.M. Carpay, J. Am. Ceram. Soc. 60 (1977) 82.

[8] C.H. Hsueh, A.G. Evans, R.L. Coble, Acta Metall. 30 (1982)1269.

[9] M.A. Spears, A.G. Evans, Acta Metall. 30 (1982) 1281.

[10] J. Svoboda, H. Riedel, Acta Metall. Mater. 40 (1992) 2829.

[11] H. Riedel, J. Svoboda, Acta Metall. Mater. 41 (1993) 1929.

[12] E. Petrishcheva, J. Renner, Acta Mater. 53 (2005) 2793.

[13] L. Klinger, E. Rabkin, L. Shvindlerman, G. Gottstein, J. Mater. Sci. 43 (2008) 5068.

[14] P. Hartland, A.G. Crocker, J. Nucl. Mater. 152 (1988) 310.

[15] N. Moelans, B. Blanpain, P. Wollants, Acta Mater. 53 (2005) 1771.

[16] N. Moelans, B. Blanpain, P. Wollants, Acta Mater. 55 (2007) 2173.

[17] K. Chang , W. Feng, L.Q. Chen, Acta Mater. 57 (2009) 5229.

[18] K. Ahmed, C. Yablinsky, A. Schulte, T. Allen, A. El-Azab, Modell. Simul. Mater. Sci. Eng. 21 (2013) 065005.

[19] K. Ahmed, J. Pakarinen, T. Allen, A. El-Azab, J. Nucl. Mater. 446 (2014) 90.

[20] K. Ahmed, T. Allen, A. El-Azab. J. Mater. Sci. 51 (2016) 1261.

[21] M. Tonks, Y. Zhang, A. Butterfield, X. Bai, Modell. Simul. Mater. Sci. Eng. 23 (2015) 045009.

[22] M. Tonks, D. Gaston, P. Millett, D. Andrs, P. Talbot, Comp. Mater. Sci. 51 (2012) 20. 35 
[23] S. Vedantam, A. Mallick, Acta Mater. 58 (2010) 272.

[24] J.W. Cahn, Acta Metall. 9 (1961) 795.

[25] M. Mahadevan, R. Bradley, Physica D 126 (1999) 201.

[26] J.W. Cahn, A. Cohen, Acta Mater. 48 (2000) 3425.

[27] C. Gugenberger, R. Spatschek, K. Kassner, Phys. Rev. E 78 (2008) 016703.

[28] S.M. Allen, J.W. Cahn, Acta Metall. 27 (1979) 1085.

[29] L. Zhang, M. Tonks, D. Gaston, J. Peterson, D. Andrs, P. Millett, B. Biner, J. Comp. Phys. 236 (2013) 74 .

[30] D. Knoll, D. Keyes, J. Comp. Phys. 193 (2004) 357.

[31] W. Mullins, J. Appl. Phys. 28 (1956) 333.

[32] L. Bourgeois, P. Dehaudt, C. Lemaignan, J.P. Fredric, J. Nucl. Mater. 295 (2001) 73.

[33] T.S. Zhang, J. Ma, L. Kong, Z.Q. Zeng, P. Hing, J.A. Kliner, Mater. Sci. Eng. B 103 (2003) 177.

[34] N. Moelans, B. Blanpain, P. Wollants, Phys. Rev. B 78 (2008) 024113.

[35] H. Emmerich, Adv. Phys. 57 (2008) 1.

[36] N. Provatas, K. Elder, Phase-Field Methods in Materials Science and Engineering. Weinheim: Wily$\mathrm{VCH} ; 2010$.

[37] Y. Wang, Acta Mater. 54 (2006) 953.

[38] S. Biswasa, D. Schwen, J. Singhc, V. Tomara, Extreme Mechanics Letters 7 (2016) 78. 

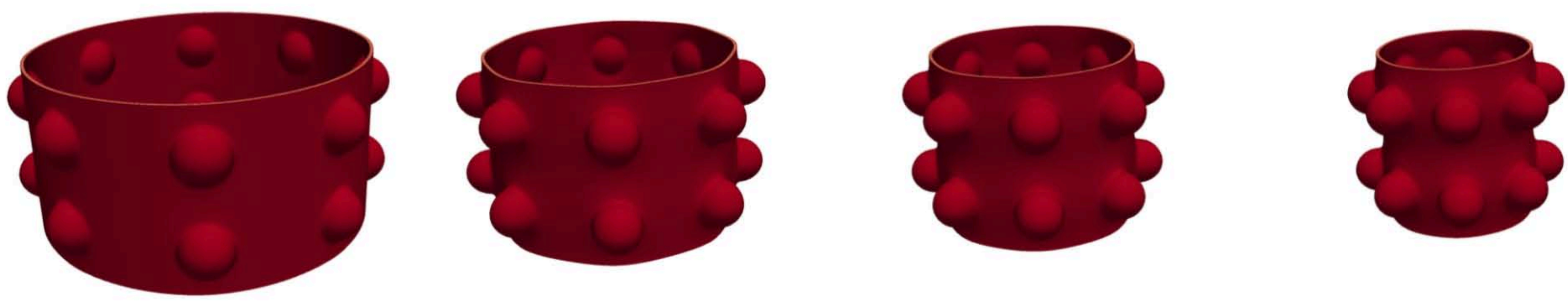

Quasi-rigid-body motion of boundary particles with a migrating grain boundary during the shrinkage of a cylindrical grain. The boundary moves under the influence of its curvature and the particles migrate via surface diffusion; particle with high surface mobility could move along with the migrating boundary as a quasi-rigid-body. 